\title{
Building PulseNet International: An Interconnected System of Laboratory Networks to Facilitate Timely Public Health Recognition and Response to Foodborne Disease Outbreaks and Emerging Foodborne Diseases
}

\author{
BALA SWAMINATHAN, ${ }^{1}$ PETER GERNER-SMIDT, ${ }^{1}$ LAI-KING NG ${ }^{2}$ \\ SUSANNA LUKINMAA, ${ }^{3}$ KAI-MAN KAM, ${ }^{4}$ SHARON ROLANDO, ${ }^{5}$ \\ ENRIQUE PÉREZ GUTIÉRREZ, ${ }^{6}$ and NORMA BINSZTEIN, ${ }^{7}$ on Behalf of All Participants \\ in the Five PulseNet Networks
}

\begin{abstract}
PulseNet USA, the national molecular subtyping network for foodborne disease surveillance, began functioning in the United States in 1996 and soon established itself as a critical early warning system for foodborne disease outbreaks, particularly those in which cases may be geographically dispersed. The PulseNet network is now being replicated in different ways in Canada, Europe, the Asia Pacific region, and Latin America. These independent networks work together in PulseNet International allowing public health officials and laboratorians to share molecular epidemiologic information in real-time and enabling rapid recognition and investigation of multi-national foodborne disease outbreaks. Routine communication between the various international PulseNet networks will provide early warning on foodborne disease outbreaks to participating public health institutions and countries.
\end{abstract}

\section{INTRODUCTION}

$\mathbf{P}$ ULSENet USA is the national molecular subtyping network for foodborne disease surveillance in the United States (Swaminathan et al., 2001). The network facilitates early recognition of foodborne disease clusters that may represent common source outbreaks. Once an outbreak is recognized, the PulseNet network provides real-time subtyping support for epidemiologists at the national, state and local (in many large counties and cities) levels. This accelerates the outbreak investigation and source identification by separating outbreak-related cases from geographically and temporally associated sporadic cases and by providing independent microbiologic confirmation of the epidemiologically implicated source.

\footnotetext{
${ }^{1}$ Foodborne and Diarrheal Diseases Branch, Division of Bacterial and Mycotic Diseases, National Center for Infectious Diseases, Coordinating Center for Infectious Diseases, Centers for Disease Control and Prevention, Atlanta, Georgia.

${ }^{2}$ Bacteriology and Enteric Diseases Program, National Microbiology Laboratory, Public Health Agency of Canada, Winnipeg, Manitoba, Canada.

${ }^{3}$ Statens Serum Institut, Copenhagen, Denmark.

${ }^{4}$ Public Health Laboratory Services Branch, Centre for Health Protection, Department of Health, Shek Kip Mei, Kowloon, Hong Kong.

${ }^{5}$ Association of Public Health Laboratories, Washington, D.C.

${ }^{6}$ Instituto Panamericano de Protección de Alimentos y Zoonosis (INPPAZ), Buenos Aires, Argentina.

${ }^{7}$ Departamento de Bacteriologia, Instituto Nacional de Enfermedades Infecciosas, ANLIS Dr. Carlos G. Malbran, Ministerio de Salud, Ciudad de Buenos Aires, Argentina.
} 
PulseNet received the prestigious "Innovations in American Government Award" in 1999. This award is given annually to those government programs that have been determined to be the most creative, innovative and proven to have accomplished their objective. The program is funded by the Ford Foundation, and is administered by the John F. Kennedy School of Government at Harvard University in association with the Council for Excellence in Government. The Innovations in American Government Program encourage their awardees to replicate their program as appropriate.

Because of the explosive increase in international food trade, foods produced and processed in one part of the world are now often consumed by people in countries that may be several thousand miles away. While this has greatly increased the variety and availability of foods at reasonable prices in many parts of the world, the world-wide distribution of foods also has increased the occurrence of international foodborne disease outbreaks. An excellent example of this is an alfalfa sprouts-associated outbreak of Salmonella Stanley infections that caused illnesses in two U.S. states (Arizona and Michigan) and Finland. Molecular subtyping allowed investigators to recognize that the outbreak may have had a common source; further investigation revealed that the alfalfa seeds used in all locations were from a Dutch shipper (Mahon et al., 1997).

As part of fulfilling the replication mandate from the Innovations Program and to address global foodborne disease concerns, PulseNet USA began an informal collaboration with their counterparts in Canada in 1999. Both sides realized the importance of this collaboration when they investigated an outbreak of shigellosis that was traced to contaminated parsley (CDC, 1999b; Naimi et al., 2003; Public Health Agency of Canada, 1999; Wu et al., 2000) in 1998 that caused illnesses in several Canadian provinces and multiple U.S. states. Canadian provincial public health departments and state health departments spearheaded the collaboration and spurred their federal coordinators into informal collaboration that continued until 2005. The informal collaboration provided valuable early warning on an international outbreak of Salmonella Oranienburg due to con- taminated chocolate produced in Germany (Werber et al., 2005), an outbreak of Salmonella Poona infections in United States and Canada in 2001 and 2002 due to cantaloupe imported from Mexico (CDC, 2002), and a Canadian outbreak of Salmonella Infantis infections caused by contaminated pig-ear dog treats processed in Texas (Clark et al., 2001; Public Health Agency of Canada, 2000).

The successful PulseNet collaboration between the United States and Canada attracted the attention of public health officials in Europe who sent laboratory personnel to the Centers for Disease Control and Prevention (CDC) for training in the PulseNet standardized pulsedfield gel electrophoresis (PFGE) protocols and software-assisted analysis of the PFGE patterns. Subsequently, CDC organized meetings in Honolulu, Hawaii (December 2002) and Buenos Aires, Argentina (December 2003) to familiarize public health officials in the Asia Pacific region and Latin America respectively with PulseNet and its role in foodborne disease surveillance. This manuscript describes the progress that has been made in establishing these international PulseNet networks, coordination of the networks, and the proposed methods of communication between them in the framework of PulseNet International.

\section{PULSENET CANADA}

PFGE has been used in many clinical, food, and reference laboratories in Canada to genotype bacterial pathogens strains since the early 1990s. The desire to compare results between laboratories in detecting outbreaks of multi-geographical regions brought a group of laboratory managers across the country to the National Microbiology Laboratory (NML) in 1998 to standardize PFGE protocols. By consensus, the laboratories developed a standardized 1day PFGE protocol for methicillin resistant Staphylococcus aureus (MRSA) and adopted the CDC protocol for Shiga-toxin producing E. coli (STEC) O157:H7. In 2000, PulseNet North was born and then renamed PulseNet Canada in 2003. The current PFGE protocols used by PulseNet Canada have been harmonized with those used by PulseNet USA. 
The provincial laboratories of the Canadian Public Health Laboratory Network (CPHLN) send weekly reports of number of laboratoryconfirmed cases of Salmonella, E. coli O157:H7, Shigella, Yersinia, Campylobacter, and parasites as part of the National Enteric Surveillance Program (NESP) (Table 1). These data provide disease trends that have been used to detect potential outbreaks by noting an unexpected high number of cases observed during a week.

\section{Organization and structure}

For preparedness and response to natural and intentional outbreaks of infectious disease, improved methods of collection and dissemination of information across different jurisdictions are required. To accomplish this objective, NML has established a virtual framework, the Canadian Laboratory Surveillance Network (CLSN), for molecular epidemiology of infectious disease agents that may have national and international impact. PulseNet Canada has been placed within this framework. CLSN is managed under the Canadian Public Health Laboratory Network. International collaborations of PulseNet Canada is established through the Public Health Agency of Canada (PHAC). Besides NML, PulseNet Canada memberships include the Laboratory of Foodborne Zoonoses (LFZ) of the Public Health Agency of Canada (PHAC), the Bureau of Microbial Hazards, Food Directorate, Health Canada, and ten provincial laboratories of the CPHLN located in British Columbia, Alberta, Saskatchewan, Manitoba, Ontario, Québec, New Brunswick, Nova Scotia, Prince Edward Island, and Newfoundland.

The strategic plan for PulseNet Canada was developed in collaboration with epidemiologists, while goals and objectives are a result of consultation with CPHLN directors and the PulseNet working group. The PulseNet working group includes the Chief of Enteric Diseases, NML and supervisors of provincial molecular typing laboratories. The working group has monthly meetings to discuss progress and resolve technical problems. Also, an Executive Steering Committee was established for PulseNet Canada with members from participating provinces. Monthly teleconferences were scheduled for discussion and updates. In 2003, an on-line national database was established for PulseNet Canada and housed in the NML with appropriate data security and data backup features. Prior to 2003, data sharing and communication was done by email. After signing an agreement between PulseNet Canada participants, members can interrogate the $E$. coli, Salmonella, Shigella, and Listeria monocytogenes database in real-time and upload patterns and data.

\section{Communication and exchange of information}

NML and provincial laboratories actively participate in PulseNet USA activities including the Annual PulseNet Meeting sponsored by

Table 1. Cases of Major Enteric Pathogen Infections in Canada, 1999-2004 (DemczuK, 2005)

\begin{tabular}{|c|c|c|c|c|c|c|}
\hline Organism & 1999 & 2000 & 2001 & 2002 & 2003 & 2004 \\
\hline Salmonella enterica & 7338 & 4510 & 6645 & 6498 & 5531 & 5525 \\
\hline E. coli $\mathrm{O} 157^{\mathrm{a}}$ & 2703 & 1835 & 1386 & 1259 & 1163 & 1013 \\
\hline Shigella spp. & 1239 & 1150 & 918 & 1360 & 935 & 747 \\
\hline Yersinia $^{\mathrm{b}}$ & 702 & 666 & 912 & 725 & 655 & 505 \\
\hline Parasites $^{\mathrm{b}}$ & - & 5673 & 6612 & 5969 & 4526 & 5534 \\
\hline Campylobacterc & 11778 & 12072 & 11892 & 11508 & 9994 & - \\
\hline
\end{tabular}

${ }^{a}$ E. coli $\mathrm{O} 157$ includes E. coli O 157 STEC, E. coli O157, E. coli O157:H7, and E. coli O157:NM isolations.

${ }^{\mathrm{b} C r y p t o s p o r i d i u m}$ and Cyclospora were not nationally notifiable until January 2000. Entamoeba and Yersinia are not notifiable, and numbers of cases of illness are those reported to the NESP and may be underreported.

'Totals of Campylobacter and parasitic infections are largely based on data supplied by the NDRS, whereas the total number of isolations of other organisms relies on NESP data. The collection of total Campylobacter infection data for 2003 and 2004 by NDRS is not complete at the time of publication.

Organism totals include number of laboratory-confirmed infections reported to the National Enteric Surveillance Program supplemented with identification data obtained through reference services provided by NML and infections reported through the National Notifiable Diseases Reporting System (NDRS). 
CDC. During the meeting, experience, success stories, and research activities are shared. Faceto-face meetings are the key to success of international PulseNet collaboration. The international PulseNet representatives collaborate and work together to overcome barriers to data sharing of information across jurisdictions. These barriers could be complex involving laboratory issues, differences in informatics platforms or policy, legal issues, and political will. NML has regular meetings with the Bureau of Microbial Hazards, Food Directorate, and the Canadian Food Inspection Agencies to improve the microbial quality of supply by setting policies and response.

\section{Multi-national outbreaks involving Canada and the United States}

The following examples are of outbreaks that affected people in Canada and the United States. Informal real-time exchange of PFGE patterns between NML and CDC facilitated the investigation and provided early warning on the outbreaks.

S. Enteritidis in mung beans, 2001. During February and March 2001, 83 cases of $S$. Enteritidis phage type (PT) 913 were reported in Alberta, British Columbia, and Saskatchewan. The PFGE data and epidemiological investigation linked the source to mung bean sprouts (Honish and Nguyen, 2001). A similar multistate outbreak involving S. Enteritidis PT913 was reported in the United States in May 2001. PFGE data sharing between the United States and Canada enabled public health officials to establish a connection between the two outbreaks.

S. Muenchen in orange juice, 1999. On June 25, 1999, a PulseNet USA listserv posting from the Washington State Public Health Department reported an unusual increase of Salmonella Muenchen infections, and eight of these cases had isolates that shared an indistinguishable PFGE pattern. Upon further investigation, 177 cases related to this outbreak were uncovered in 15 states (CDC, 1999a). The source of this outbreak was identified as commercially distributed unpasteurized orange juice from a sin- gle processor. Cases associated with the outbreak were also identified in Canada. The Alberta Provincial Laboratory identified four clinical isolates that matched the outbreak PFGE pattern (Public Health Agency of Canada, 1999a). The British Columbia Centre for Disease Control identified 19 temporally associated cases of $S$. Muenchen infections in that province; eight patients reported drinking orange juice that was traced back to the single processor identified above (Buxton et al., 1999).

S. Enteritidis in Almonds 2000/2001 and 2003/ 2004. A 2000-2001 outbreak of $S$. Enteritidis was first recognized due to the increase in strains of a rare phage type 30 (PT30) in Ontario, Nova Scotia, and New Brunswick in late December. Further investigation led to the identification of 157 cases in five Canadian provinces; $93 \%$ of the affected individuals who were interviewed recalled eating almonds in the 5 days preceding their illness. The Ontario Provincial Laboratory was able to isolate $S$. Enteritidis from the implicated almonds. This resulted in a national recall, after which, new cases of $S$. Enteritidis PT30 dropped dramatically. PFGE patterns of the Canadian PT30 strains were compared with PFGE patterns of American strains to identify strains related to this outbreak (Chan et al., 2002); 11 cases were identified in five states (Isaacs et al., 2005). The contaminated almonds were traced back to a single processor in California. Alerts were distributed by the World Health Organization to increase the awareness of almonds contamination (International Food Safety Authority Network, 14 June 2004 (〈www.who.int/foodsafety/fs_management/ en/infosan_salm.pdf $\rangle)$.

The experience from the 2000-2001 almond outbreak allowed investigators in the United States to quickly recognize and investigate another almond-associated outbreak in 2004. On May 12, 2004, the Oregon Public Health Laboratory identified a cluster of five patients infected with $S$. Enteritidis isolates that were indistinguishable from each other by PFGE using two different restriction enzymes. An additional 24 patients with SE of the same PFGE profile were identified in 12 states through PulseNet USA (CDC, 2004); unlike the previous outbreak, most of the affected people in this 
outbreak were in the United States. Only one case was identified by PulseNet Canada. On May 18, 2004, the processor of the implicated almonds recalled 13 million pounds of the product. The rapid detection and investigation of this outbreak and the recall of the implicated product only 6 days after the recognition of the outbreak averted many additional cases of illness from the contaminated product. In addition to distribution in North America, the processor had exported the almonds to France, Italy, Japan, Korea, Malaysia, Mexico, Taiwan, and the United Kingdom.

\section{Impact of PulseNet}

Outbreak investigations initiated from PulseNet Canada clusters have facilitated risk assessments and resulted in consumer education campaigns, improvement in industrial food processing practices, and improvements in the microbiological quality of imported foods. In some cases, other sources of outbreaks due to enteric pathogens were linked to nonfood sources such as petting zoos (Helwig, 2000; Public Health Agency of Canada, 2004), pet treats, and recreational water parks (Gilbert et al., 2005). The Public Health Agency of Canada (PHAC) is working with provincial partners to develop policies for minimizing exposure and risks from these sources.

\section{Applied research}

The NML is currently participating in a CDC validation study of multi-locus variable number tandem repeat analysis (MLVA) for subtyping E. coli O157:H7. In addition, NML is engaged in applied research to extend the MLVA primer sets to other STEC serotypes, including O26:H11 and O111:NM.

\section{Challenges for PulseNet Canada}

Formal agreements will be required to share information and integrate information from the different databases in the Canadian public health system. Currently, there is agreement for sharing information of limited scope between the NML and other laboratories in Canada. In August 2005, a memorandum of understanding was signed between the United
States and Canada to enable real-time sharing of information about clinical isolates and their subtypes between NML and CDC.

\section{PULSENET EUROPE}

In the late 1980s and early 1990s, the number of cases of salmonellosis increased dramatically in Europe and it was recognized that infections and their sources might be linked in different countries. The Salm-net project was initiated in 1994 under the leadership of the Public Health Laboratory Service, now the Health Protection Agency (HPA), in the United Kingdom. The aims of Salm-net were to harmonize the Salmonella phage-typing schemes used in Europe and to establish a timely database containing data on Salmonella infections throughout Europe (Fisher, 1995). The participants were the public health reference institutions in the European Union (EU), Norway, and Switzerland. Later, Australia, Canada, Japan, and South Africa joined the network. In 1997, STEC O157:H7 was added to the system, which changed its name to Enter-net (Fisher, 1999). The database was soon expanded to include antimicrobial susceptibility data in addition to data on the sero- and phage type of the isolates (Threlfall et al., 1999). Besides the database, an email-based rapid alert system moderated by the project leader was set up so that one participant could alert all other participants about national outbreaks with possible international connections. That system has been very successful in delineating and investigating international outbreaks (Anonymous, 1994, 1997; Crook et al., 2003; Fisher, 1997; Werber et al., 2005). However, by the end of the 1990s, it was evident that more discriminatory subtyping systems were needed for outbreak investigations. A PulseNet Europe working group was established within Enter-net to determine the feasibility of introducing the American PulseNet model in Europe. At the same time an EU-funded research project, Salm-gene (2001-2004), was undertaken among nine participants of Enter-net under the leadership of HPA (Fisher and Threlfall, 2005; Peters et al., 2003). The objectives of this project were to harmonize the PFGE protocols between the 
participants and subtype human Salmonella isolates initially retrospectively and later prospectively and submit the raw images of the PFGE profiles to HPA, where they were entered into a customized BioNumerics database. The project proved the principle set by PulseNet USA that it should be possible to compare PFGE patterns of Salmonella generated in different public health laboratories, also in Europe. At the conclusion of the project, the Salm-gene database contained approximately 20,000 PFGE patterns mainly of the serotypes Typhimurium and Enteritidis. During the same period, a need for a more active surveillance of listeriosis (Lister-net) (de Valk et al., 2003) and the need for cross-disciplinary surveillance of foodborne zoonosis (〈www.cost920.com $\rangle$ ) were identified. A group of scientists from Enter-net, Lister-net and the COST 920-action decided to initiate a feasibility study for molecular surveillance of Salmonella, Shiga toxin-producing E. coli, and Listeria monocytogenes to ascertain the interest in such a network among veterinary and public health reference laboratories in Europe and to test the skills of the future participants in performing PFGE using a protocol compatible with the one used by PulseNet USA. The study was a success and some of the results are described elsewhere in this issue of the journal (Gerner-Smidt et al., 2006). PulseNet Europe was founded as a result of this initiative.

\section{Organization and structure}

In 2004, PulseNet Europe received funding for 18 months to establish the core infrastructure as a work package in the FP6 (FP6-2002FOOD-1-506122) network of excellence, MedVet-Net (〈www.medvetnet.org $\rangle), \quad$ a virtual European Zoonosis Centre. PulseNet Europe was organized in September 2004, with a project coordinator and a Steering Committee (Fig. 1). The partners are 57 institutes from 30 countries; these include 35 public health institutes, 21 veterinary or food institutes, and one institute which receives both public health and veterinary isolates (Fig. 2).

The current objectives in PulseNet Europe are to establish a real-time surveillance database system for food, public health and veterinary laboratories, to detect clusters of similar DNA fingerprints, and to investigate outbreaks of Salmonella, STEC and Listeria monocytogenes, to set up a central database and a rapid communication system for partners, and to train

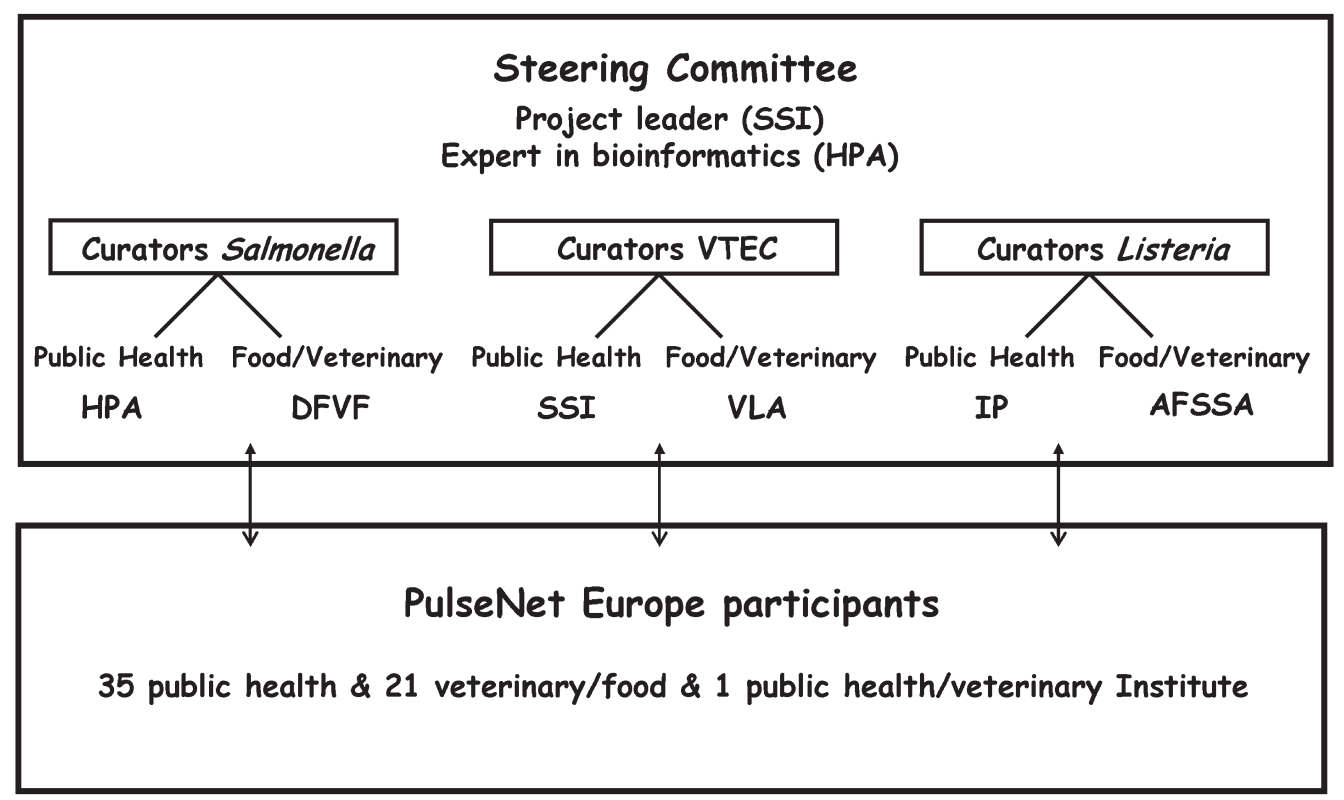

FIG. 1. PulseNet Europe organization structure. SSI, Statens Serum Institut, Denmark; HPA, Health Protection Agency, England; DFVF, Danish Institut for Food and Veterinary Research, Denmark; VLA, Veterinary Laboratories Agency, England; IP, Institut Pasteur, France; AFSSA, French Food Safety Agency, France. 


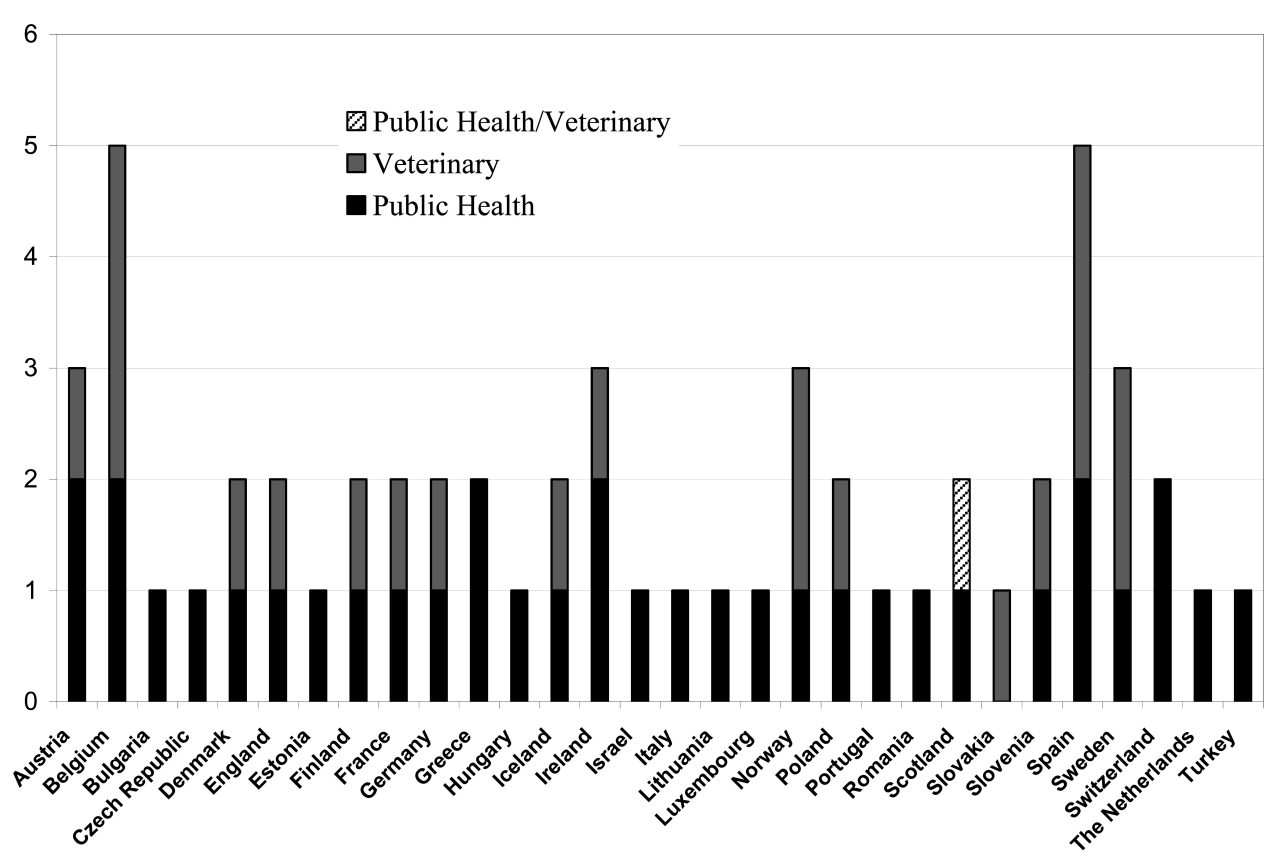

FIG. 2. Participating countries and institutes in PulseNet Europe.

database curators and partners. A memorandum of understanding will be executed with the other international PulseNet networks to enable real-time exchange of subtyping and other important public health information to enable rapid tracking of foodborne disease outbreaks at the global level.

The PulseNet Europe database is built in the BioNumerics server/client format that is compatible with the other databases in PulseNet International and the on-line database server is placed at the Health Protection Agency, United Kingdom. Six PulseNet Europe database curators from England, Denmark, France, and an expert in bioinformatics from England have online access to the central database and are responsible for maintaining the database (Fig. 2). This includes assuring quality of the data, validating, and naming the PFGE profiles submitted by partners. The curators will also be responsible for performing central cluster analyses and alerting the participants and epidemiologists when a cluster of PFGE profiles are detected.

\section{Activities}

As of September 2005, six curators have been trained in the use of the central database. Also in 2005, PulseNet Europe created a homepage ( $\langle$ www.pulsenet-europe.org $\rangle$ ) and a prototype of a closed but unmoderated listserv where the members may post rapid alerts about clusters of infections and discuss other subjects pertinent to PulseNet.

The funds from Med-Vet-Net are not aimed at training participants or sustaining and developing the database. However, PulseNet Europe applied for extra funding for training of partners. The funding was granted and in November 2005, 14 partners will be trained to perform PFGE and image analysis according to rules defined by PulseNet Europe. The PulseNet Europe database currently only contains data from the Salm-gene project and national data from the curator countries and it will not be open for the participants before they have been trained and certified.

\section{Challenges for PulseNet Europe}

The present funding for PulseNet Europe will end in February 2006, and the biggest challenge is to find alternative funding to build and maintain PulseNet Europe. How to position PulseNet Europe in the current surveillance systems in Europe is another big challenge. A possibility would be to integrate the existing 
surveillance systems for foodborne infections, especially Enter-net and PulseNet Europe under the same umbrella under the auspices of the newly founded European Centers for Disease Control (Ekdahl, 2005). However, at present, Enter-net participants are only from the public health sector and the Enter-net databases contain information only about infections caused by Salmonella, STEC, and most recently Campylobacter. In contrast, PulseNet has members from both the public health sector and the food and veterinary sectors and deals with Listeria monocytogenes as well. By combining a real-time surveillance within the cross-disciplinary platform and broad microbial coverage of PulseNet with the strong infrastructure and experience in Enter-net, it would be possible to build a strong surveillance system for foodborne infections in Europe.

\section{PULSENET ASIA PACIFIC}

Soon after the first planning meeting held in Honolulu in December 2002, PulseNet Asia Pacific was officially established with a network of members from the public health laboratories in the region with a common goal of facilitating the timely exchange of DNA fingerprinting data of foodborne pathogens. The consortium has now drawn together 13 countries and areas (Australia, Bangladesh, Hong Kong, India, Japan, Korea, Malaysia, New Zealand, Philippines, Peoples's Republic of China, Taiwan, Thailand, and Vietnam) with a combined estimated population (2001) of 2.9 billion.

\section{Organization and structure}

A Steering Committee consisting of representatives from Australia, Bangladesh, China, Hong Kong, Japan, Malaysia, Philippines, and Taiwan with Dr. Kai Man Kam, Public Health Laboratory Centre, Hong Kong acting as chair/coordinator, develops and approves a strategic vision and plan for PulseNet Asia Pacific. Individual country and area representatives are urged to formulate action plans for establishing PulseNet activities in their own country/area. Communications take place mainly through emails, but the possibility of establishing a threaded email platform for rapid exchanges has been discussed and explored.

\section{Activities}

The PulseNet Asia Pacific Steering Committee conducted a survey to identify the importance of individual pathogens to the participating countries/areas, and the following foodborne pathogens were listed in decreasing order of priority: Salmonella (including S. Typhi and non-typhoidal salmonellae), E. coli O157:H7, Vibrio cholerae, Shigella spp., Vibrio parahaemolyticus, Campylobacter spp., and Listeria monocytogenes. This list formed the basis for mutual understanding and planning of further activities.

The Second PulseNet Asia Pacific Meeting was held March 18-19, 2004, at the Public Health Laboratory Centre in Hong Kong. Delegates shared their progress and experiences in building PFGE capability and setting up infectious disease surveillance network within their countries. Possible approaches to obtain funding from international bodies, as well as options and strategies to raise awareness of policy-makers were also discussed as a method to increase national funding. Training was identified as another important key to the success of PulseNet Asia Pacific. Work Groups were formed to tackle the major issues in the region through the provision of platforms for new initiatives, free discussion, exchange of information, and coordinated research in specific areas/topics. The Work Groups formed at present are (1) Laboratory Resource and Support (LRS); (2) Salmonella Subtyping (SS); (3) Server Development (SD); and (4) Platform for Inter-laboratory Comparison (PIC).

In the PIC Work Group Phase I comparison, five isolates of Salmonella were sent by the coordinating laboratory to the participating laboratories. The resulting TIFF images were sent back to the coordinating laboratory for comparison. These sample strains were distributed to seven countries/areas in the region, and facilitated the comparison of results using PulseNet standardized protocols. Recently, the LRS Work Group was able to secure support 
from the Japan Ministry of Health and Welfare. This will ensure a sustained build-up of laboratory capacity to perform standardized PFGE in the country/area that can benefit from this funding.

\section{Intra-country networks}

Several countries in the Asia Pacific region have already established or are in the process of establishing national PulseNet networks. The oldest and best established network is Pulse-Net Japan which is part of the Wide-area Information-exchange System for Health, Labor and Welfare administration, and coordinated by the National Institute for Infectious Diseases (NIID). Like PulseNet USA, PulseNet Japan began working with STEC O157 and is currently expanding coverage to other pathogens, including non-O157 STEC, Salmonella, Shigella, and Vibrio. NIID is presently collaborating with prefectures and municipal public health institutes on PulseNet Japan activities and is actively seeking collaborations with institutes in the Ministry of Agriculture, Forestry and Fisheries to further facilitate tracing of outbreaks to their food sources.

PulseNet Aotearoa (= land of the long white cloud; native Maori name) New Zealand was established in 2004 by the Institute of Environmental Science and Research Limited, a government-owned research institute that includes New Zealand's public health and enteric reference laboratories. The initial focus of the network will be on Campylobacter spp., Salmonella, Listeria, Shigella, and STEC. Human Campylobacter infections are particularly serious public health problems in New Zealand due to their high incidence with notified rates approaching 400/100,000. Standardized PFGE protocols have been established for all four pathogens, and a national server has been established with databases for the four pathogens. The Campylobacter database contains PFGE patterns of 1126 isolates, $91 \%$ of which are C. jejuni.

The Division of Enteric Infections, Korea Center for Disease Control and Prevention began building PFGE capacity for epidemiologic investigations in 1997 and has embarked on building a national PulseNet network. At present, 10 provincial institutes of Health and En- vironment participate in PulseNet Korea and are able to perform standardized subtyping of Salmonella and Shigella. Other countries in this region are actively involved in establishing molecular subtyping capacity and building real-time subtyping network. Most notably, the People's Republic of China has made a strong commitment to establishing a national molecular subtyping network for foodborne disease surveillance. PulseNet China was officially inaugurated in September 2004, is coordinated by the China Center for Disease Control, and has already recruited 26 provincial centers for disease controls for participation in the network.

Since 2000, the National Institute of Cholera and Enteric Diseases in India with funding from the Japan International Cooperation Agency has disseminated a standardized PFGE protocol for enteric pathogens to many Indian laboratories.

\section{Outbreak investigations}

Collaboration across borders can be seen in real life events that occurred in the region. In February 2004, the Okinawa Prefectural Chubu Health Center and the Okinawa Prefectural Institute of Health and Environment, Japan, investigated E. coli O157:H7 infections in a Japanese family associated with eating ground beef (CDC, 2005). Exchange of PFGE patterns between PulseNet Japan and PulseNet USA led to the identification of cases with the same unique PFGE pattern in the western United States. Public health officials from multiple agencies in Japan and the United States collaborated on this investigation, which resulted in a voluntary recall of approximately 90,000 pounds of frozen ground beef in the United States and at American military bases in the Far East. This was the first reported instance in which Japanese public health officials identified contaminated, commercially distributed ground beef that was produced in the United States. The results underscore the importance of using standardized molecular subtyping methods throughout the world to facilitate international public health communication and intervention.

In September 2005, the Japanese Ministry of Health informed the Hawaii Department of 
Health that seven airline passengers returning from Hawaii had been infected with Shigella sonnei. Further investigation accompanied by exchange and comparison of PFGE patterns between PulseNet Japan and PulseNet USA allowed public health officials to ultimately identify more than 40 infections from four countries (United States, Japan, Australia, and Samoa) associated with flights originating from Honolulu in late August 2004. The most likely vehicle for this outbreak was salad served in airline meals prepared by the caterer (Gaynor and Kate, personal communication).

PFGE subtyping enabled investigators to recognize a new clone of multidrug-resistant Shigella dysenteriae type 1 that has emerged in the Indian subcontinent and has caused outbreaks and sporadic disease. This strain is resistant to ampicillin, co-trimoxazole, tetracycline, chloramphenicol, nalidixic acid, norfloxacin, lomefloxacin, pefloxacin, ofloxacin, and showed reduced susceptibility to ciprofloxacin (Pazhani et al., 2004).

\section{Research}

Three PulseNet Asia Pacific laboratories (Bangladesh, Japan, and Hong Kong) are collaborating with PulseNet USA in the development and validation of a standardized PFGE protocol for Vibrio cholerae (Cooper et al., 2006). An international set of Vibrio cholerae strains was assembled for this project by the participants. It is envisaged that availability of a standardized PulseNet PFGE protocol for Vibrio cholerae will greatly enhance the global surveillance of this pathogen that has high public health significance with the potential for use as a biothreat agent.

\section{Challenges of PulseNet Asia Pacific}

The development of PulseNet Asia Pacific faces many challenges. The pace and volume of trade, as well as outbreaks, has been increasing rapidly in the region. These outbreaks have occurred within the diverse cultural and socio-economic background of the different countries/areas. A number of places also suffered from limited penetration of leaders down to working levels. The lack of financial, as well as human, resources is also prominent espe- cially among public health laboratories serving less developed areas. Enhancement of mutual understanding in the use of standardized PFGE protocols will form a firm basis for future developments. As such, the Platform for Inter-laboratory Comparison (PIC) Work Group attempts to fill this gap. In time, it is hoped that trust and understanding will develop between the different participants.

Since different countries/areas differ in their progress in development of PFGE and their action plan, lessons learned can be more easily shared to quicken the steps of laboratory enhancement in less developed areas. In particular, laboratory capacity building in terms of training, problem solving, supervision, and quality controls are prominent issues that must be addressed. Appropriate training and expertise of the participants form the cornerstone for all other activities. Since the steering committee has identified the pathogens, which are of common interest to the participant laboratories, a stepwise approach to collaborate in efforts to tackle each of these is pertinent to the common interests of all.

\section{PULSENET LATIN AMERICA}

Reliable data on foodborne disease outbreaks in Latin America and the Caribbean is scarce. Countries in this region reported less than 7,000 outbreaks in the past 10 years to the WHO/PAHO Regional Information System on foodborne diseases surveillance-SIRVETA (〈www.panalimentos.org/sirveta $\rangle$ ). Countries in the region have different public health systems resulting in wide variations in the level of reporting of diseases. For foodborne diseases, one of the major causes of insufficient data is that the majority of the countries are collecting syndrome data (e.g., diarrhea or food poisoning), with or without laboratory confirmation. There is no formal laboratory-based surveillance system.

To remedy this weakness, the Pan American Health Organization (PAHO/WHO) has developed an initiative based on the promotion of networking to improve and/or strengthen the countries' capabilities in food monitoring and surveillance of foodborne disease. The 
overall goal of the initiative is to have integrated food-chain surveillance systems established along the region. As part of the $\mathrm{PAHO} / \mathrm{WHO}$ networking initiative, PulseNet Latin America was established in partnership with CDC and the National Institute of Infectious Diseases of Argentina, the "Dr. Carlos G. Malbrán" Institute. The network presently has participation from 13 countries, and its mission is to strengthen the surveillance of foodborne diseases in Latin America through the application of standardized molecular subtyping techniques.

\section{Organization and structure}

The network was established at a consultation meeting held in Buenos Aires in December 2003, with the participation of microbiologists and epidemiologists from 13 Latin American countries. The participants agreed to build PulseNet Latin America and developed a plan of action for the incorporation of PulseNet into countries' surveillance systems. A Regional International Steering Committee was designated and the bylaws of the network discussed. The bylaws were approved by country members on July 10, 2004 (〈www.panalimentos.org/pulsenet $\rangle$ )

The organizational structure of the PulseNet Latin America is composed of the following:

1. Assembly of Members: The members are the designated institutions and/or laboratories of each participating country. Each country is represented by a Technical Contact from the designated institution/laboratory in PulseNet Latin America. The Member is responsible for operating the information system and for communication within PulseNet Latin America and at a national level, the Member coordinates PulseNet training and certification, and proficiency testing. The Assembly is the highest authority in the network within the boundaries of its expertise. To date, two general assemblies have been held resulting in the approval of the action plans for PulseNet Latin America.

2. The Technical Coordination Unit is comprised of $\mathrm{PAHO} / \mathrm{WHO}, \mathrm{CDC}$ and the Malbran Institute. The Assembly of members may designate additional representatives to the unit. The principal duties of the Technical Coordination Unit are training, research, and communication. PAHO is the trustee of the regional data bank.

3. The International Steering Committee is composed of one microbiologist and one epidemiologist from each of four member countries of PulseNet Latin America elected by the Assembly. Their duties are to represent PulseNet Latin America at the meetings of the PulseNet International, promote the interaction of PulseNet Latin America with other organizations and to elaborate with the technical coordinators a plan of action and a budget in a biennial basis.

\section{Objectives and action plan implementation}

The first objective of PulseNet Latin America was to implement standardized PFGE methodology for the subtyping of Salmonella spp. and Escherichia coli O157 in participating institutions, and standardized analysis of the PFGE patterns using BioNumerics. PulseNet Latin America organized and conducted two comprehensive training courses covering both aspects for microbiologists from the member institutions in 2004 and 2005. Participants from the National Reference Laboratories from Argentina, Brazil, Chile, Colombia, Mexico, Uruguay, Venezuela, Costa Rica, Nicaragua, Bolivia, Paraguay, Cuba, and Peru were trained. At the end of both training courses, the participants were supplied with the PulseNet reference Salmonella Braenderup strain and some critical reagents to facilitate the rapid implementation of the standardized PFGE protocols in their respective laboratories. Also, the members of PulseNet Latin America have agreed on a standardized pattern naming system, minimum data that will be submitted with the PFGE patterns, and a certification and proficiency testing program. Also, the Malbrán Institute conducted an assessment of PFGE subtyping capabilities of participating laboratories by sending seven (Salmonella) isolates to participating laboratories and evaluating the quality of the PFGE patterns submitted.

The regional databases for PulseNet Latin America will be located on a server at the Pan 
American Institute for Food Protection and Zoonosis (INPPAZ) in Argentina. Network hardware acquisition and maintenance, compliance with information security standards, and providing controlled server access to participants will be responsibility of INPPAZ. The Malbrán Institute will initially assume curator responsibility for the PulseNet Latin America databases. As additional pathogen databases are set up on the server, curator responsibilities for the new databases may be assumed by other participants.

A website has been developed for PulseNet Latin America to facilitate communication between members (〈www.panalimentos.org/ pulsenet $\rangle)$. The website also has a listserv to improve communications between members, and an electronic forum to facilitate discussions is being developed.

\section{Challenges of PulseNet Latin America}

Lack of political support is a major issue in the consolidation of the network. Also, all member countries must improve disease detection and notification of outbreaks, institute active surveillance for selected pathogens, and initiate burden of illness studies. Communication between the food production and the public health sectors needs to be improved.

PulseNet Latin America will continue to emphasize an integrated foodborne disease surveillance system in Latin America. Several activities have been planned for implementation at the country level and regional level during the next 24 months to achieve this objective. At the country level, efforts will be focused on providing support to the laboratories in each country. The members of PulseNet Latin America will aggressively promote further training in workshops, training courses and promote political commitment, within the plan of action developed with input from the Assembly of Members. Also, PulseNet Latin America will foster the development of within-country laboratory networks that integrate food and human aspects. National training courses will be organized on the identification and characterization of the prioritized pathogens within each country and information flow from the laboratory and disease surveillance will be stream- lined. At the regional level, PulseNet Latin America will conduct training in the diagnosis and standardized subtyping of pathogens prioritized on the basis of their regional prevalence.

\section{CONCLUSIONS}

PulseNet International is a network of PulseNet national and regional networks. The PulseNet International objectives are to facilitate a global foodborne disease surveillance, an early recognition of international foodborne disease outbreaks, and a collaborative approach to outbreak investigations; to delineate the geographical distribution and dynamics of important clones of foodborne pathogens; and to provide information that may assist the participating networks in establishing policies for safer food within and outside of their regions.

PulseNet International is governed by the PulseNet International Steering Committee, which has coordinating officials of the participating networks as members. The committee, which is chaired by CDC, will provide guidance, and make recommendations about the work and the strategic development of the network. It meets face-to-face at least once annually and will convene conference calls as necessary.

The work in Pulsenet International has so far concentrated on establishing the infrastructure of each network as described in this paper. Although laboratories in all PulseNet networks perform PFGE on a variety of foodborne pathogens, only PulseNet USA and Canada have functional databases accessible to all participants for Shiga toxin-producing E. coli, Salmonella, Shigella, Listeria monocytogenes and Campylobacter. This situation will change during the next year with servers being established in all regions.

The different approaches used to establish each international PulseNet network reflect the differences in the political, economical, and organizational structures in the regions. Despite major political, economic, and administrative impediments, significant advances have been made during the past 5 years toward building a PulseNet International network. The formal 
Memorandum of Understanding executed in 2005 between Canada and the United States is a major step forward in inter-country exchange of real-time information on foodborne disease clusters and outbreaks. This agreement will be a model for the development of similar agreements between other international PulseNet networks. After these agreements are executed, each PulseNet network will be able to log on to the server of any other international PulseNet network as a client (with read-only access to clinical isolate information in its pathogen databases) and query the databases for matches to pathogen subtypes of interest, and, if matches are found, access the epidemiologic information on matching isolate patterns. There are currently no plans to establish a central server containing all information from all international PulseNet networks.

Each network will eventually have access to the following information about the isolates in the databases of the other networks: source country, isolation date of the specimen (if available), the date the isolate was received in the laboratory performing the subtyping (if available), the date the pattern of the isolate was uploaded to the regional PulseNet server, the serotype of the isolate (if available), and the PFGE patterns of the isolate. If one PulseNet network during an investigation of an outbreak detects a matching pattern in the database of a sister PulseNet network, the coordinator of the sister network will be contacted and asked to participate in the investigation. The first connection between two network databases is scheduled to be established between PulseNet Canada and PulseNet USA in 2006.

CDC and the Association of Public Health Laboratories (APHL) have been catalysts in the establishment of the international PulseNet networks. Until now, this global expansion of PulseNet has not received adequate funding to support its objective and mission. Fortunately, advances have been made through the enthusiasm, commitment, and dedication of the public health officials of participating countries. Some countries (e.g., Japan, People's Republic of China, New Zealand) have recognized the potential of PulseNet and have allocated significant resources to establish PulseNet-compatible networks within their countries. Re- search grants recently awarded from the National Institute of Infectious Diseases, Japan to 11 member institutions of PulseNet Asia Pacific to enhance their laboratory capacity for molecular subtyping and to facilitate their active participation in the network are particularly gratifying. The European Union has provided partial funding for the establishment of PulseNet Europe but the positioning of the network in the surveillance of foodborne infections needs to be defined in that region. Several countries in PulseNet Latin America and Caribbean urgently need funding to enhance their foodborne disease surveillance and laboratory capabilities and to begin active participation in this global food safety network.

\section{DISCLAIMER}

Use of trade names is for identification only and does not imply endorsement by the Centers for Disease Control and Prevention or by the U.S. Department of Health and Human Services.

\section{REFERENCES}

Anonymous. 1994. A foodborne outbreak of Shigella sonnei infection in Europe. Commun Dis. Rep. CDR Wkly. 4:115.

Anonymous. 1997. European collaboration identifies an outbreak of Escherichia coli infection in visitors to Fuerteventura, Canary Islands. Commun. Dis. Rep. CDR Wkly. 7:127.

Buxton, J., M. Fyfe, A. King, A. Paccagnella, K. Campbell, A. Ellis, and J. Hockin. 1999. Outbreak of Salmonella serotype Muenchen infection in the United States and Canada associated with unpasteurized orange juice-the British Columbia experience. Can. Commun. Dis. Rep. 25:161-164.

CDC (Centers for Disease Control and Prevention). 1999a. Outbreak of Salmonella serotype Muenchen infections associated with unpasteurized orange juice-United States and Canada, June 1999. MMWR 48:582-585.

CDC (Centers for Disease Control and Prevention). 1999b. Outbreaks of Shigella sonnei infection associated with eating fresh parsley-United States and Canada, July-August 1998. MMWR 48:285-289.

CDC (Centers for Disease Control and Prevention). 2002. Multistate outbreaks of Salmonella serotype Poona infections associated with eating cantaloupe from MexicoUnited States and Canada. MMWR 51:1044-1047.

CDC (Centers for Disease Control and Prevention). 2004. Outbreak of Salmonella serotype Enteritidis infections as- 
sociated with raw almonds-United States and Canada, 2003-2004. MMWR 53:484-487.

CDC (Centers for Disease Control and Prevention). 2005. Escherichia coli O157:H7 infections associated with ground beef from a U.S. military installation-Okinawa, Japan. MMWR 54:40-42.

Chan, E.S., J. Aramini, B. Ciebin, D. Middleton, R. Ahmed, M. Howes, I. Brophy, I. Mentis, F. Jamieson, F. Rodgers, M. Nazarowec-White, S.C. Pichette, J. Farrar, M. Gutierrez, W.J. Weis, L. Lior, A. Ellis, and S. Isaacs. 2002. Natural or raw almonds and an outbreak of a rare phage type of Salmonella Enteritidis infection. Can. Commun. Dis. Rep. 28:97-99.

Clark, C., J. Cunningham, R. Ahmed, D. Woodward, K. Fonseca, S. Isaacs, A. Ellis, C. Anand, K. Ziebell, A. Muckle, P. Sockett, and F. Rodgers. 2001. Characterization of Salmonella associated with pig-ear dog treats in Canada. J. Clin. Microbiol. 39:3962-3968.

Cooper, K.L.F., C.K.Y. Luey, M. Bird, J. Terajima, G.B. Nair, K.M. Kam, E. Arakawa, A. Safa, D.T. Cheung, C.P. Law, H. Watanabe, K. Kubota, B. Swaminathan, and E. Ribot. 2006. Development and validation of a PulseNet standardized pulsed-field gel electrophoresis protocol for subtyping of Vibrio cholerae Foodborne Pathog. Dis. 3:51-58.

Crook, P.D., J.F. Aguilera, E.J. Threlfall, S.J. O' Brien, G. Sigmundsdottir, D. Wilson, I.S. Fisher, A. Ammon, H. Briem, J.M. Cowden, M.E. Locking, H. Tschape, W. van Pelt, L.R. Ward, and M.A. Widdowson. 2003. A European outbreak of Salmonella enterica serotype Typhimurium definitive. Clin. Microbiol. Infect. 9:839-845.

de Valk, H., C. Jacquet, V. Goulet, V. Vaillant, A. Perra, J.-C. Desenclos, P. Martin, and the Listeria Working Group. 2003. Feasibility study for a collaborative surveillance of Listeria infections in Europe. Report to the European Commission. DG-Sanco, Paris, France. Available at: 〈www.invs.sante.fr/publications/2004/listernet/〉.

Ekdahl, K. 2005. Senior appointments and new facilities announced as the ECDC is inaugurated. Eur. Surveill. Available at: (www.eurosurveillance.org/ew/2005/ 050602.asp\#5>.

Fisher, I.S. 1995. Salm-Net: A network for human Salmonella surveillance in Europe. Eur. Surveill. 0:7-8. Available at: 〈www.eurosurveillance.org/em/v00n00/0000-225.asp〉.

Fisher, I.S. 1997. An international outbreak of Salmonella livingstone recognised by Enter/Salm-net. Eur. Surveill. Available at: 〈www.eurosurveillance.org/ew/1997/ 971218.asp $\rangle$.

Fisher, I.S. 1999. The Enter-net international surveillance network-how it works. Eur. Surveill. 4:52-55.

Fisher, I.S., and E.J. Threlfall. 2005. The Enter-net and Salm-gene databases of foodborne bacterial pathogens that cause human infections in Europe and beyond: an international collaboration in surveillance and the devel- opment of intervention strategies. Epidemiol. Infect. 133:1-7.

Gerner-Smidt, P., and F. Scheutz. On Behalf of the Study Participants. 2006. Standardized pulsed-field gel-electrophoresis of Shiga toxin-producing Escherichia coliThe PulseNet Europe feasibility study. Foodborne Pathog. Dis. 3:9-19.

Gilbert, M., L. Srour, A. Paccagnella, L. MacDougall, J. Fung, E. Nelson, and M. Fyfe. 2005. An outbreak of Escherichia coli O157:H7 associated with a children's water spray park and identified by two rounds of pulsed-field gel electrophoresis testing. Can. Commun. Dis. Rep. 31:133-140.

Helwig, D. 2000. E. coli outbreak linked to fall fair. Can. Med. Assoc. J. 162:245.

Honish, L., and Q. Nguyen. 2001. Outbreak of Salmonella enteritidis phage type 913 gastroenteritis associated with mung bean sprouts-Edmonton, 2001. Can. Commun. Dis. Rep. 27:151-156.

Isaacs, S., J. Aramini, B. Ciebin, J.A. Farrar, R. Ahmed, D. Middleton, A.U. Chandran, L.J. Harris, M. Howes, E.S. Chan, A.S. Pichette, K. Campbell, A. Gupta, L.Y. Lior, M. Pearce, C. Clark, F. Rodgers, F. Jamieson, I. Brophy, and A. Ellis and the Salmonella Enteritidis PT30 Outbreak Investigation Working Group. 2005. An international outbreak of salmonellosis associated with raw almonds contaminated with a rare phage type of Salmonella Enteritidis. J. Food Prot. 68:191-198.

Mahon, B.E., A. Ponka, W.N. Hall, K. Komatsu, S.E. Dietrich, A. Siitonen, G. Cage, P.S. Hayes, M.A. LambertFair, N.H. Bean, P.M. Griffin, and L. Slutsker. 1997. An international outbreak of Salmonella infections caused by alfalfa sprouts grown from contaminated seeds. J. Infect. Dis. 175:876-882.

Naimi, T.S., J.H. Wicklund, S.J. Olsen, G. Krause, J.G. Wells, J.M. Bartkus, D.J. Boxrud, M. Sullivan, H. Kassenborg, J.M. Besser, E.D. Mintz, M.T. Osterholm, and C.W. Hedberg. 2003. Concurrent outbreaks of Shigella sonnei and enterotoxigenic Escherichia coli infections associated with parsley: implications for surveillance and control of food-borne illness. J. Food Prot. 66:535-541.

Pazhani, G.P., B. Sarkar, T. Ramamurthy, S.K. Bhattacharya, Y. Takeda, and S.K. Niyogi. 2004. Clonal multidrug-resistant Shigella dysenteriae type 1 strains associated with epidemic and sporadic dysenteries in Eastern India. Antimicrob. Agents Chemother. 48:681-684.

Peters, T.M., C. Maguire, E.J. Threlfall, I.S. Fisher, N. Gill, and A.J. Gatto. 2003. The Salm-gene project-a European collaboration for DNA fingerprinting for food-related salmonellosis. Eur. Surveill. 8:46-50.

Public Health Agency of Canada. 1999a. Outbreak of Salmonella serotype Muenchen infections associated with unpasteurized orange juice-United States and Canada, June 1999. Can. Commun. Dis. Rep. 25:161-164. 
Public Health Agency of Canada. 1999b. An outbreak of Shigella sonnei in British Columbia-September 1998. Can. Commun. Dis. Rep. 25:73-78.

Public Health Agency of Canada. 2000. Human health risk from exposure to natural dog treats. Can. Commun. Dis. Rep. 26:41-42.

Public Health Agency of Canada. 2004. Petting zooassociated Escherichia coli O157:H7-secondary transmission, asymptomatic infection, and prolonged shedding in the classroom. Can. Commun. Dis. Rep. 30:173-179.

Swaminathan, B., T.J. Barrett, S.B. Hunter, and R.V. Tauxe. 2001. PulseNet: the molecular subtyping network for foodborne bacterial disease surveillance in the United States. Emerg. Infect. Dis. 7:382-389.

Threlfall, E.J., I.S. Fisher, L.R. Ward, H. Tschape, and P. Gerner-Smidt. 1999. Harmonization of antibiotic susceptibility testing for Salmonella: results of a study by 18 national reference laboratories within the European
Union-funded Enter-net group. Microb. Drug Resist. 5:195-200.

Werber, D., J. Dreesman, F. Feil, U. van Treeck, G. Fell, S. Ethelberg, A.M. Hauri, P. Roggentin, R. Prager, I.S. Fisher, S.C. Behnke, E. Bartelt, E. Weise, A. Ellis, A. Siitonen, Y. Andersson, H. Tschape, M.H. Kramer, and A. Ammon. 2005. International outbreak of Salmonella oranienburg due to German chocolate. BMC Infect. Dis. 5:7.

Wu, F.M., M.P. Doyle, L.R. Beuchat, J.G. Wells, E.D. Mintz, and B. Swaminathan. 2000. Fate of Shigella sonnei on parsley and methods of disinfection. J. Food Prot. 63:568-572.

Address reprint requests to: Dr. Bala Swaminathan Centers for Disease Control and Prevention 1600 Clifton Rd., Mailstop C03 Atlanta, GA 30333 E-mail: bas5@cdc.gov 